\title{
Neuromodulation via Interferential Electrical Stimulation as a Novel Therapy in Gastrointestinal Motility Disorders
}

\author{
Judith S Moore, ${ }^{*}$ Peter R Gibson and Rebecca E Burgell \\ Department of Gastroenterology, Central Clinical School, Monash University and Alfred Hospital, Melbourne, Victoria, Australia
}

The concept of therapeutic percutaneous neuromodulation has, until recently, been limited by the ability to penetrate deeply enough to stimulate internal organs. By utilizing 2 medium frequency, slightly out of phase electrical currents passing diagonally through the abdomen, a third, low frequency current is created at the point of bisection. This interferential current appears to stimulate nerve fibers in the target organs and may have a therapeutic action. The aim of the study is to review the use of transcutaneous interferential electrical stimulation with a focus on its application in gastroenterology, particularly in motility disorders. Studies involving use of interferential current therapy were searched from Medline, PubMed, and Scopus databases, and articles pertaining to history, its application and all those treating abdominal and gastrointestinal disorders were retrieved. Seventeen studies were identified, 13 involved children only. Eleven of these were randomised controlled trials ( 3 in adults). Four trials were from the one center, where each paper reported on different outcomes such as soiling, defecation frequency, quality of life, and colon transit studies from the one pool of children. All studies found statistically significant improvement in symptom reduction. However, weaknesses in study design were apparent in some. In particular, finding an adequate placebo to interferential current therapy has been difficult. Interferential current therapy shows potential as a novel, non-pharmacological and economical means of treating gastrointestinal dysfunction such as constipation. More studies are needed particularly in the adult population. However, the design of a suitable placebo is challenging.

(J Neurogastroenterol Motil 2018;24:19-29)

Key Words

Constipation; Electrical stimulation; Gastroenterology

\section{Introduction}

Since the discovery of electricity, it has been known that nerve fibers can be stimulated by an electrical current. ${ }^{1}$ Electrical therapy was first utilized in Europe for neurological stimulation in the 19th century and possibly earlier in the Orient. More recently, neuromodulation has been in use clinically for the last 20 years, predomi- nantly treating pain, tremor, or spasticity. Its use has now grown and neuromodulation has been employed in many novel applications across cardiology, neurology, psychiatry, urology, and not least, gastroenterology. Given the proposed pathogenic mechanisms for functional gastrointestinal disorders (alteration of efferent or afferent function of the enteric and extrinsic nervous system), neuromodulation has increasingly gained traction for treating functional gastrointestinal symptoms resistant to conservative treatments. The best

Received: June 9, 2017 Revised: November 1, 2017 Accepted: December 2, 2017

(c) This is an Open Access article distributed under the terms of the Creative Commons Attribution Non-Commercial License (http://creativecommons. org/licenses/by-nc/4.0) which permits unrestricted non-commercial use, distribution, and reproduction in any medium, provided the original work is properly cited.

*Correspondence: Judith S Moore, RN MN

Department of Gastroenterology, Alfred Hospital, Level 6, The Alfred Centre, 99 Commercial Rd, Melbourne, Victoria 3004, Australia Tel: +61-3-9903-0233, Fax: +61-3-9903-0556, E-mail: Judith.moore@monash.edu 
studied of these is the application of sacral neuromodulation (commonly known as sacral nerve stimulation) for fecal incontinence. ${ }^{2}$

Sacral nerve stimulation is delivered via a percutaneous transforaminal approach, with the electrical current delivered directly to the sacral nerve roots known to control the pelvic viscera. ${ }^{3}$ Sacral nerve stimulation has been shown to reduce episodes of fecal incontinence and improve quality of life. ${ }^{4}$ It has also been trialled as a treatment for constipation with less impressive outcomes. ${ }^{5}$ However, this method is expensive and invasive, and requires surgical procedures under general anesthetic. ${ }^{6}$ Given this, sacral nerve stimulation is not recommended as a first-line management strategy. Other noninvasive and more economical neuromodulatory techniques have thus been explored, such as pudendal nerve stimulation, ${ }^{7}$ posterior tibial nerve stimulation, ${ }^{8,9}$ and magnetic stimulation of the sacral nerves. ${ }^{10}$ This review will address the latest technique, transcutaneous interferential current therapy.

\section{Search Method}

A search of Medline, PubMed, and Scopus databases for information on electrical interferential current and its application using search words such as "electrical interferential current," "transcutaneous," "therapeutics or uses," "mechanisms of action," and "gastrointestinal" was performed. Articles that were available in English were included. This review focuses on the use of transcutaneous interferential current therapy in the treatment of gastrointestinal symptoms.

\section{The Nature of Interferential Current Therapy}

Interferential current therapy has been used in a variety of settings including low back pain ${ }^{11}$ and neurological disorders such as carpal tunnel syndrome. ${ }^{12}$ More recently, interferential current therapy has been found to be effective in small studies in managing conditions such as fecal incontinence and constipation. It has the benefit of being completely non-invasive, cost effective and convenient, as it can be self-administered at home. ${ }^{13}$

Despite increased convenience to the patient, doctor and payer, the use of transcutaneously-delivered current can be problematic. To stimulate the nerve of interest, the current has to pass through the skin and surrounding muscle to reach its target. This causes resistance to flow, requiring the use of a higher current to achieve the desired result. Skin impedance is inversely proportional to the frequency of the current. ${ }^{14}$ Low frequency currents, which are needed to stimulated nerves, result in high skin resistance. To overcome this, a higher current is needed, which can cause pain. In contrast, high frequency currents results in low skin resistance and passes through without pain. ${ }^{15}$ Unfortunately, these currents are not suitable to stimulate nerves. Interferential current therapy hopes to overcome this dilemma. An interferential current is produced when 2 medium-frequency alternating currents that are slightly out of phase are crossed. ${ }^{16}$ This new modulated current is believed to be produced at the site of bisection of the 2 diagonally opposed currents by their interference with each other. ${ }^{17}$ The use of interferential current therapy dates back to the 1950s where it was found that the use of an interferential current overcame or bypassed skin impedance that is usually experienced with low frequency currents, but allowed the benefits of a low frequency current to occur due to the development of an amplitude-modulated current within the target of interest. ${ }^{18,19}$

It has been demonstrated that an interferential current can reach targeted deeper tissue if the target tissue lies on a diagonal path between the circuits outside the electrode border. ${ }^{17}$ However, in reality, interferential stimulation is likely more complex. Interestingly, despite the growing popularity of interferential current therapy in various clinical settings, there have been few studies on its efficacy or its dispersion through body tissues.

The situation is further complicated by the fact there are 2 types of interferential currents: the "true" interferential current that is generated by the use of 4 electrodes and the "premodulated" interferential current that is generated within a device that delivers the currents and transmits via 2 electrodes only. ${ }^{17}$ As would be expected, the true interferential current as opposed to the premodulated interferential current had the greater voltage recording at depth, showing superiority in efficiency of stimulation at deeper levels. ${ }^{17,20}$ With the "true" interferential current, the orientation and location of nerve fibers in relation to the electrodes affects whether the nerve fibers experience unmodulated or continuous, fully modulated, or partially modulated stimuli. ${ }^{21}$ Orientation of the fibers along a current pathway results in zero modulation. More efficient modulation occurs in contrast, if fibers are oriented at the point of bisection of the stimulation axes. ${ }^{20}$ How this translates to the stimulation of frequently mobile intra-abdominal structures is unknown. "True" interferential current therapy is believed to have its maximal stimulation deep at the intersection of the 2 currents whereas premodulated is thought to act superficially near the electrodes. Consequently, it is the "true" interferential current that is recommended for abdominal or deep tissue use. ${ }^{19}$

A $4 \mathrm{kHz}$ carrier frequency for the 2 currents has been found to 
more effective than 8 or $10 \mathrm{kHz}$ in producing a hypoalgesic effect in back pain, and it is this setting that is frequently used in a variety of scenarios for interferential current therapy. ${ }^{19,22}$ At this frequency, it is claimed that there is better penetration through to deeper tissue while overcoming the problem of skin impedance.

\section{Potential Actions of Interferential Current Therapy on Various Systems}

The mechanism of action of interferential current therapy in gastrointestinal disorders is not understood. Whether it is via stimulation of the interstitial cells of Cajal, of the pacemaker cells of the gut, or of the enteric or extrinsic autonomic nerves is still to be defined. However, as the given effects of stimulation are not immediate and last for some months after stimulation had ceased, they are more likely to be driven by alteration of neuronal function rather than by directly causing myogenic contractions. ${ }^{23}$ It is postulated that an interferential current may influence the neuroplasticity of the enteric nerves, inducing structural, intrinsic or synaptic changes leading to altered neuronal function. Certainly neuroplasticity has been associated with motility disturbances in inflammatory bowel disease and irritable bowel syndrome. ${ }^{24}$

Interferential current causes increased propagating sequences and increased colonic activity, ${ }^{23}$ but evidence has yet to be found to determine the precise mechanism of action. One hypothesis is that interferential stimulation exerts its effects via electrically stimulating excitable cells such as the interstitial cells of Cajal, which produce slow wave activity in the bowel responsible for peristalsis, or that it directly stimulates the nerves of the enteric nervous system. ${ }^{22} \mathrm{Al}-$ ternatively, because the placement of electrodes is in close proximity to the spinal cord, it is also suggested that its effects may be exerted directly to the spinal cord, influencing the autonomic (or extrinsic) nervous system either through the afferent or efferent pathways. It is feasible that, when used for bladder dysfunction, the sinusoidal current created by the interferential currents acted on the spinal cord around T12-L1. ${ }^{25}$

There is also the possibility that hormonal systems are affected to explain the lingering effects after treatment. Increased endogenous levels of gamma-aminobutyric acid and opioid agonists may also be induced by interferential current stimulation promoting antispastic effects for a short time. ${ }^{26}$ This is supported by a case report of a patient taking concurrent tramadol who displayed the effects of opioid use such as drowsiness, decreased alertness and inability to concentrate for several hours after treatment by interferential current therapy. ${ }^{27}$ It was hypothesized that interferential current therapy stimulated production of endorphins or enkephalins and that the additive effect of tramadol triggered this response. However, these theories have yet to be confirmed and indeed a placebo response has not been adequately excluded.

The placebo effect associated with the therapeutic alliance that develops with some practitioners is frequently a confounder in assessing the therapeutic effect of such an intervention. In a study of patients with chronic lower back pain, active and sham interferential current therapy were compared in conjunction with either a limited (minimal interaction with the therapist administering the treatment) or an enhanced therapeutic interaction with participants (active listening, demonstrating concerns) to examine the placebo effect. ${ }^{11}$ The strongly positive response to active therapy with enhanced interaction was encouraging however the greater benefit of sham interferential current therapy with enhanced interaction over the real interferential current therapy and limited interaction was also enlightening. Certainly, a powerful influence of the therapeutic alliance was demonstrated ${ }^{11}$ and future studies of this technique must consider the role of placebo. For instance, in a randomised controlled study of children with juvenile arthritis, half of the study group had combined interferential current therapy and resistive underwater exercises where the other half, as the control group, received traditional physical therapy alone and no underwater therapy. ${ }^{28}$ The authors suggested that improvements in muscle strength and pain reduction was due to interferential current therapy theorised to be due to its actions on the local blood supply and suppression of paininducing chemicals. However, there is no direct evidence of this and it is entirely possible that the underwater therapy alone could have produced the same results; it was after all a novel therapy for these children, water being both less painful in which to move and more fun. Hence, this positive outcome may also have been secondary to the placebo response.

Interferential current therapy has been tested against placebo in studies examining the pain response in healthy subjects ${ }^{29}$ where interferential current therapy is known to significantly increase the pain threshold. It has been reported as a safe therapeutic option for a number of conditions with a variety of actions including analgesia, vasodilatation, and anti-inflammatory and sympatholytic effects. In addition it may also stimulate circulation and promotes a decrease in interstitial edema as suggested by a study examining its use in carpal tunnel syndrome. ${ }^{12,30}$ However, only 2 studies included robust objective measures such as electroneurophysiological indicators (sensory nerve conduction velocity) in carpal tunnel syndrome, ${ }^{12}$ passive range of movement in hemiplegic shoulders, ${ }^{30}$ and improved balance and gait in stroke victims. ${ }^{20}$ 
The application of interferential current therapy for urological conditions has been studied in both adult and pediatric settings. ${ }^{31,32}$ Several studies have explored the effects of interferential current therapy on urodynamic measures and continence in children with myelomeningocele and detrusor overactivity, and in non-neuropathic underactive bladder. These studies showed normalization of bladder function. Interestingly, in these studies it was noted that diarrhea was a side effect sometimes being reported the day after commencing therapy. It was this finding that led to interest in interferential current therapy as a treatment for bowel dysfunction. ${ }^{33}$

\section{Gastrointestinal Application}

\section{Studies in children}

The majority of studies on the application of transcutaneous interferential current therapy for constipation have arisen from Melbourne, Australia where several studies on children with slowtransit constipation have been conducted. ${ }^{13}$ Chronic constipation remains a difficult condition to treat and children refractory to traditional treatments face surgical procedures such as appendicostomy or colectomy.

Results from the first pilot study on interferential current therapy in children were published in $2005^{9}$ with findings supporting a "proof-of-concept" that neuromodulation via interferential therapy improved colonic function. Eight children with severe constipation, of whom 7 had significant soiling problems, were treated via a physiotherapist. Three children had appendicostomies. The application of interferential current therapy to the lower gastrointestinal tract was via placement of 2 electrodes on the abdomen lateral to the umbilicus and 2 on the back at the level of T9-L2. Leads were connected from the right front to the left back and vice versa so that the currents crossed (Fig. 1). ${ }^{13}$ Participants received between 9 and 12 stimulation sessions over a 4-week period. ${ }^{9}$ Soiling disappeared in the first 2 weeks after interferential current therapy in 6 of the 7 , although long-term effects were less impressive. Constipation was considered resolved in 7 of the 8 children.

The success of this pilot study led to other studies over the next few years with children randomised to receive actual or placebo treatment. Three studies involving children from this larger randomized controlled study reported on different outcome measures. The effects of interferential current therapy on propagating sequences was reported on a sub-group of 7 children who had an appendicostomy and were able to undergo 24-hour colonic manometry. These children had an increase in the frequency of antegrade propagating contractions with manometric patterns improving to

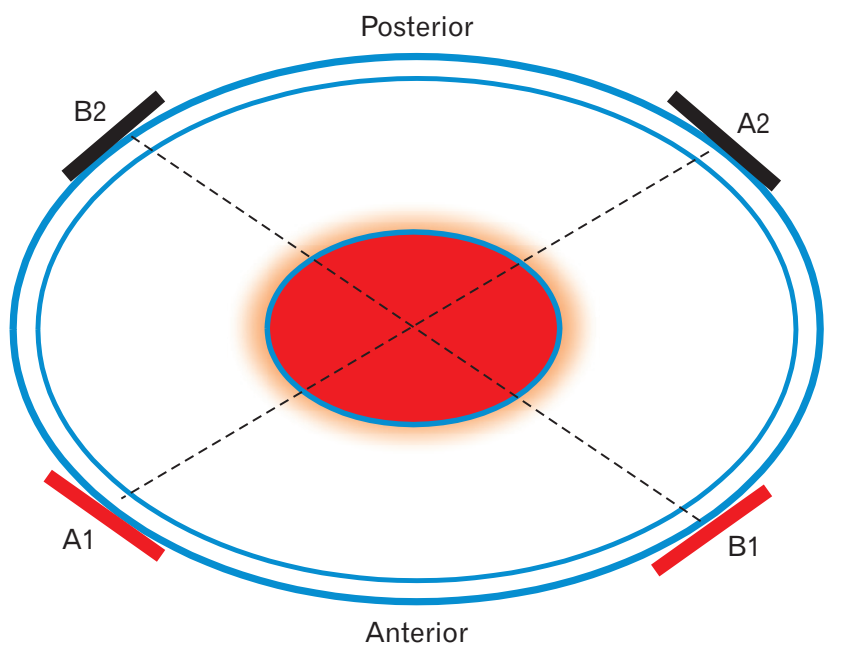

Figure 1. Pathway of interferential currents showing the positioning of electrodes on the abdomen and the creation of a third current at point of bisection inside the abdomen.

lie in the normal range. ${ }^{13,33}$ Nuclear transit studies in some of these same children showed an improvement in transit time. However, those with concurrent upper gastrointestinal dysmotility did not respond as well to interferential current therapy compared with those participants with slow transit colon and normal upper gastrointestinal motility. ${ }^{34}$ As a response gradually appeared over time rather than instantly, it is possible that the improvement in colonic transit times and increased propagating sequences reflect induction in changes to the neuronal circuits rather than causing direct contractions of intestinal muscle during stimulation. ${ }^{33}$

Subsequent to these early studies, and with the advent of small, hand-held interferential devices (Fig. 2), parents and children could be taught how to use the device at home. As a result, studies with larger groups of children, randomized to a longer, more frequent placebo or real stimulation (such as an hour a day daily for 3 to 6 months) were conducted. ${ }^{35,36}$ Outcome measures over these different studies included soiling, defaecation frequency, urge to defaecate, quality of life and objective transit studies. Again there was a significant improvement in varying degrees to all, though quality of life indicators showed a modest, but statistically significant improvement as reported by the children, but not by their respective parents. $^{37}$

There are some concerns with the quality of the single institution studies discussed above. The main concern is that the reporting of different outcomes on the same group of children across several different studies creates an artificial impression that the number of studies conducted is higher than it is, and it is difficult to tease out 

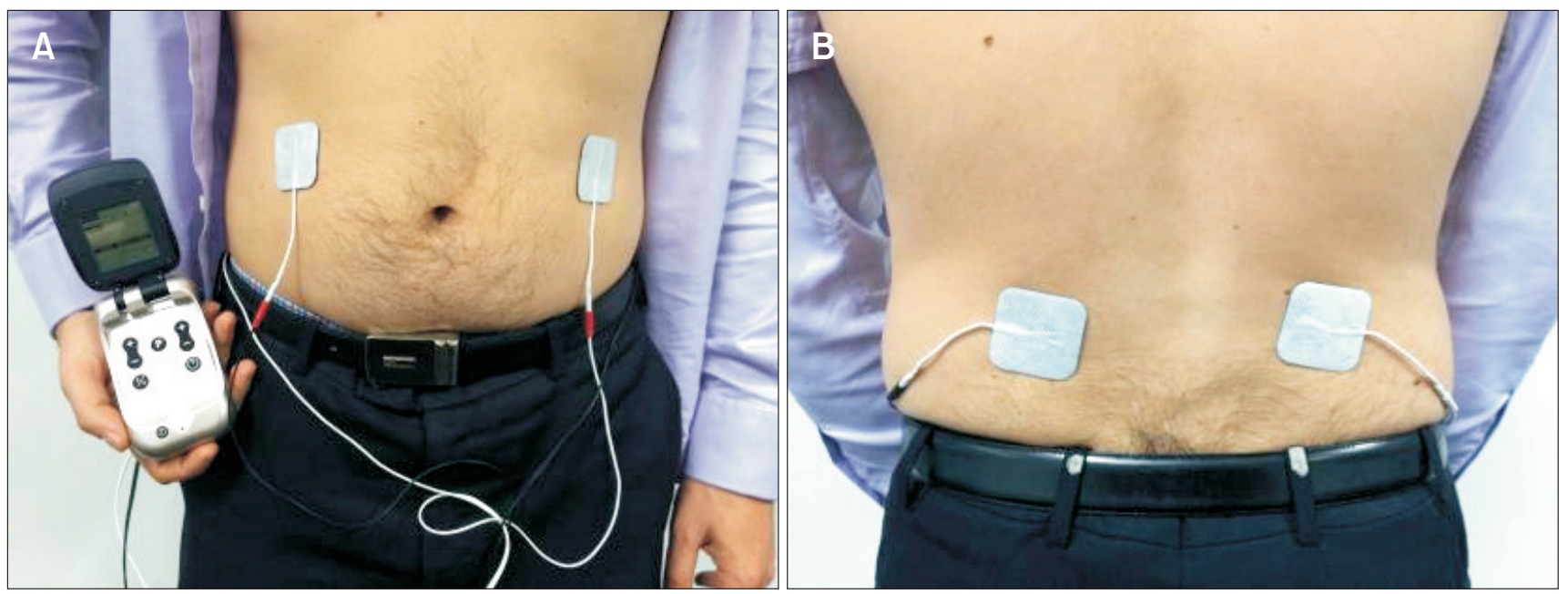

Figure 2. Position of electrodes on the abdomen and back with the hand held device for home stimulation. (A) Anterior electrodes: between costal margin and umbilicus. (B) Posterior electrodes: paraspinal between T9-L2.

whether the overall outcome measures are met.

Nevertheless, the technique has been investigated further in other centers with some success. Thirty Iranian children with neuronal bowel dysfunction from myelomeningocele participated in a randomized controlled trial. ${ }^{38}$ Constipation, as determined by stool form, decreased and the neurogenic bowel dysfunction scores improved from 'moderate' to 'mild' in the interferential current therapy group. These results were noted at 6 months after receiving treatment with an overall improvement in $73 \%$ of the children in the interferential current therapy group. In this group, it was suggested that the therapeutic action maybe more than just improved colonic transit time, and it was postulated that pelvic floor muscle fibers may be strengthened or that there may be neuromodulation of the sacral reflexes as seen in patients with urinary and fecal incontinence. ${ }^{38}$ Anorectal manometric indices also improved with a significant reduction in both sphincter pressure and the recto-anal inhibitory reflex, although it is not clear what the clinical significance of this is, as normal reference ranges are still debated. ${ }^{39}$

Two recent studies have explored the efficacy of interferential stimulation in conditions related to anal sphincter function. One study hypothesized that placement of electrodes over the lower abdomen and sacral nerves would better assist with outlet obstruction or defecatory dysfunction. Intriguingly, not only was there an increase in defecation frequency and reduction in fecal incontinence in the majority of children, but the 2 with delayed gastric emptying had a demonstrated decrease in gastric emptying time..$^{40}$ This would suggest an effect in an organ that lies outside the area of stimulation. This may be because of the heterogeneity of biological tissue affecting the pattern of current flow, ${ }^{19}$ the effects on the autonomic nervous system overall or mediated via enteroenteric reflexes. In children post-surgery for Hirschsprung disease, interferential treatment plus behavioural therapy was more successful in normalizing stool form, reducing incidences of fecal incontinence and increasing frequency of defecation than behavioural therapy alone. ${ }^{41}$ Nevertheless, success may well be attributed to the placebo effect. Use of the device with a sham current would be ideal, but a true sham stimulation has yet to be described.

Very little has been done in exploring the use of interferential stimulation in the treatment of other gut dysmotility disorders in children. As discussed before, children with slow-transit constipation and normal upper gut transit responded to interferential current therapy, whereas those with concurrent upper gastrointestinal dysmotility did not. More recently, delayed gastric emptying was improved in 2 children with delayed gastric emptying and constipation associated with rectal or colonic distension rather than slow transit. ${ }^{45}$ Formal randomized controlled trials studies have yet to be conducted.

Concurrent bladder and bowel dysfunction in children improved with additional interferential current therapy compared to education, diaphragmatic breathing exercises and behavioural modification alone. ${ }^{42}$ This observation supports the notion that electrical stimulation enhances the function of both sympathetic and parasympathetic nerve fibers including the sacral nerves, since bowel and bladder are similarly innervated. ${ }^{41}$ 


\section{Studies in adults}

A handful of studies have explored interferential current therapy in adults with constipation, but there are no reports for gastroparesis. In a French pilot study, 11 patients with proven slow transit constipation used interferential current therapy at home for an hour a day for 3 months. ${ }^{43}$ Primary outcome measures were the number of bowel motions a week and validated constipation questionnaires completed before and after the 3-month period. Colon transit studies were repeated at the end of the 3 months. Seven of the eleven significantly improved in all scores and there was a slight improvement in colonic transit times. Pre-stimulation, the majority had a median number of stools per week of 0.33 or 0.66 , and the highest post-stimulation frequency was 1.66 stools per week. Though clinically significant, these results might be regarded as sub-optimal. In addition, as there was no control group, it is possible that the improvement could be attributed to the placebo effect, particularly since the greatest improvement was in quality of life, a subjective outcome measure. As there was a quantitatively small, though statistically significant, improvement in colonic transit time, it was suggested that parasympathetic nerve fibers were being stimulated. ${ }^{43}$ It is noted that this group have a protocol published for a future randomized control study ${ }^{44}$ to follow on from this preliminary study. The results will be awaited with interest.

The second study was a randomized control trial in 28 women who met at least 2 of the 6 Rome II criteria for constipation. ${ }^{45}$ All treatments were performed by a therapist for 20 minutes a day, 3 times a week for 4 weeks. The placebo intervention $(\mathrm{n}=14)$ appeared to receive the same treatment as the therapeutic arm ( $\mathrm{n}=$ 14) with the exception that the stimulator was not actually switched on (ie, they received no current). Overall, the average number of defecations per week improved from 3.7 to 5.6 in the treatment arm, but not in the placebo group. Stool form or the number of complete defecations per week was not captured. ${ }^{46}$ There tended to be greater improvement in the constipation assessment scale and pain scores, which improved in both groups consistent with a placebo response. The authors do note the lack of objective measures as a limitation, as well as the small sample size. ${ }^{45}$ Another 2 studies were reported by a Turkish research group exploring the use of vacuum interferential current therapy in both adults with irritable bowel syndrome or functional dyspepsia. Both were randomized, blinded, placebo-controlled trials. Sham stimulation was the absence of any current, but suction cups were placed according to the study design, and still connected to the stimulating machine in the same way as active therapy, but not switched on. ${ }^{47,48}$ In both studies, treatment was administered by a physiotherapist 3 times a week for 4 weeks.
Unfortunately, neither study had physiological outcome measures, but were restricted to symptom and quality of life scales. A strong placebo response was noted in both studies with no clear difference between the significant improvements from baseline in the real or placebo arms. ${ }^{47}$ There was also a moderate participant dropout rate, possibly related to the exclusion of rescue medication. In addition, each study was at risk of responder bias as the questionnaires were administered by the study physiotherapists. Furthermore, the method of applying the electrodes in a quadripolar method on the back, rather than the abdomen and back for those with functional dyspepsia, does not fit with the suggestion that the area for treatment should be on a diagonal path where the currents cross at right angles. ${ }^{19}$ In this study, their leads were all on the one plane, which contrasts to previous reports of the transabdominal approach where 2 electrodes are on the abdomen and 2 on the back, and the currents cross diagonally through the abdomen. ${ }^{9,13}$ This may have affected the outcomes. As the results were presented as the number of patients who reported improvement, not as individual scores, the overall effect size is not clear. A further recent study on the application of transcutaneous electrical stimulation to the sacral nerves for constipation also applied electrodes on the same plane but over the sacral region. ${ }^{49}$ It is entirely possible that their failure to achieve significant effects also related to the lack of intra-abdominal cross currents. A summary of all studies on interferential current therapy and the gastrointestinal system are in the Table.

\section{Discussion}

The majority of studies into interferential therapy for fecal incontinence and constipation have been conducted at a single center only and, while results from the Melbourne group appear as though many children have been studied, a number of reports are from the same group of children. ${ }^{23,33,34}$ It is plausible that this could also be the same for studies arising from $\operatorname{Iran}^{32,38}$ as it is unclear whether the same group of children were the subjects of at least 2 of the papers. There were some differences in study design such as home stimulation for some children or physiotherapist-administered stimulation in hospital, or a variety of outcome measures. While this did not appear to impact on results of the various studies, it is clear that an evolutionary pattern in the use of interferential current therapy in children is observed as it is becoming more accepted in practice. Overall, it was noted that the benefits lasted from between 6 months and 2 years, ${ }^{50}$ and children who have since relapsed have gone on to continue home stimulation with ongoing benefit. A previous review of studies on children in Melbourne suggested that more placebo- 


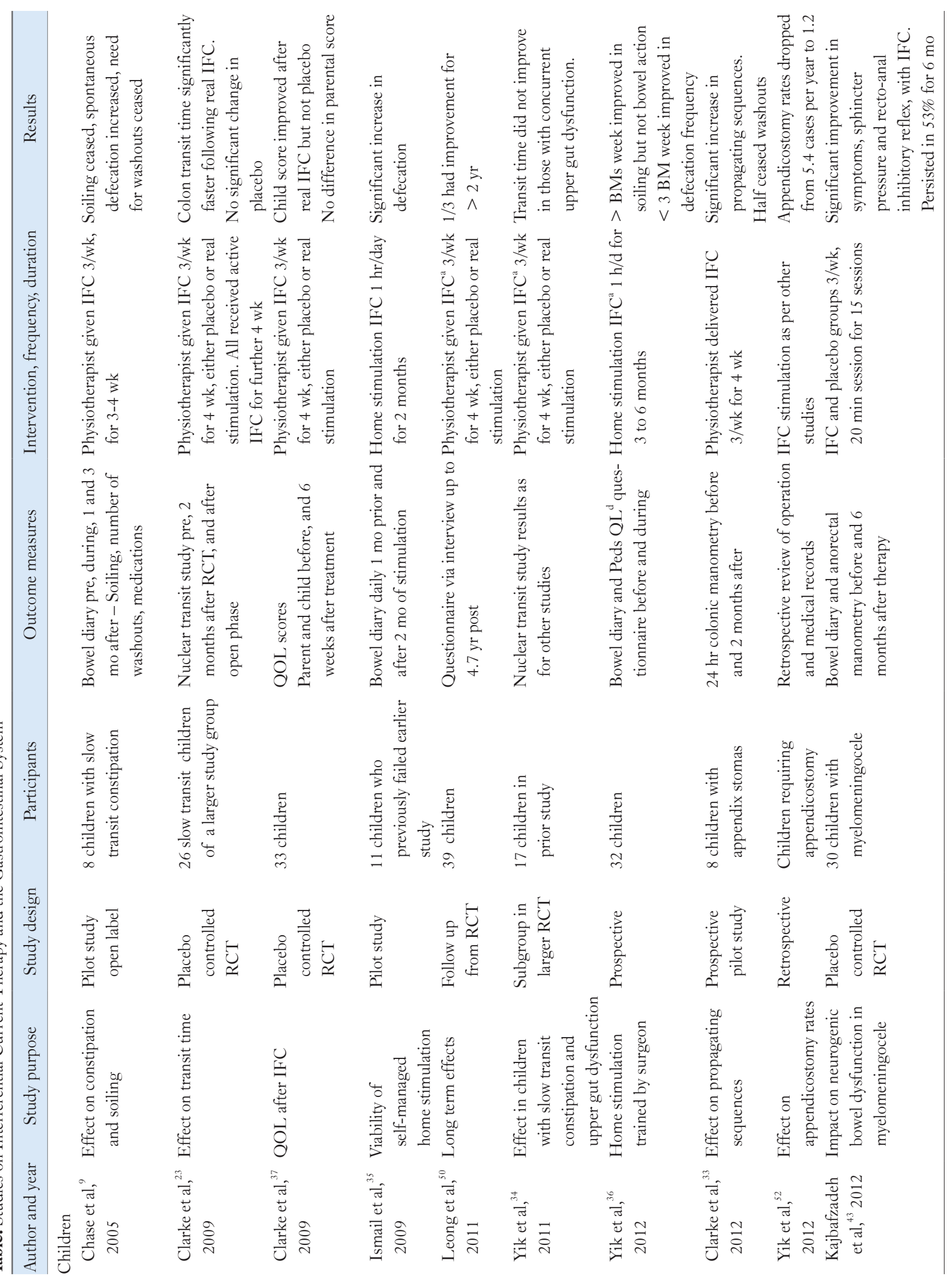




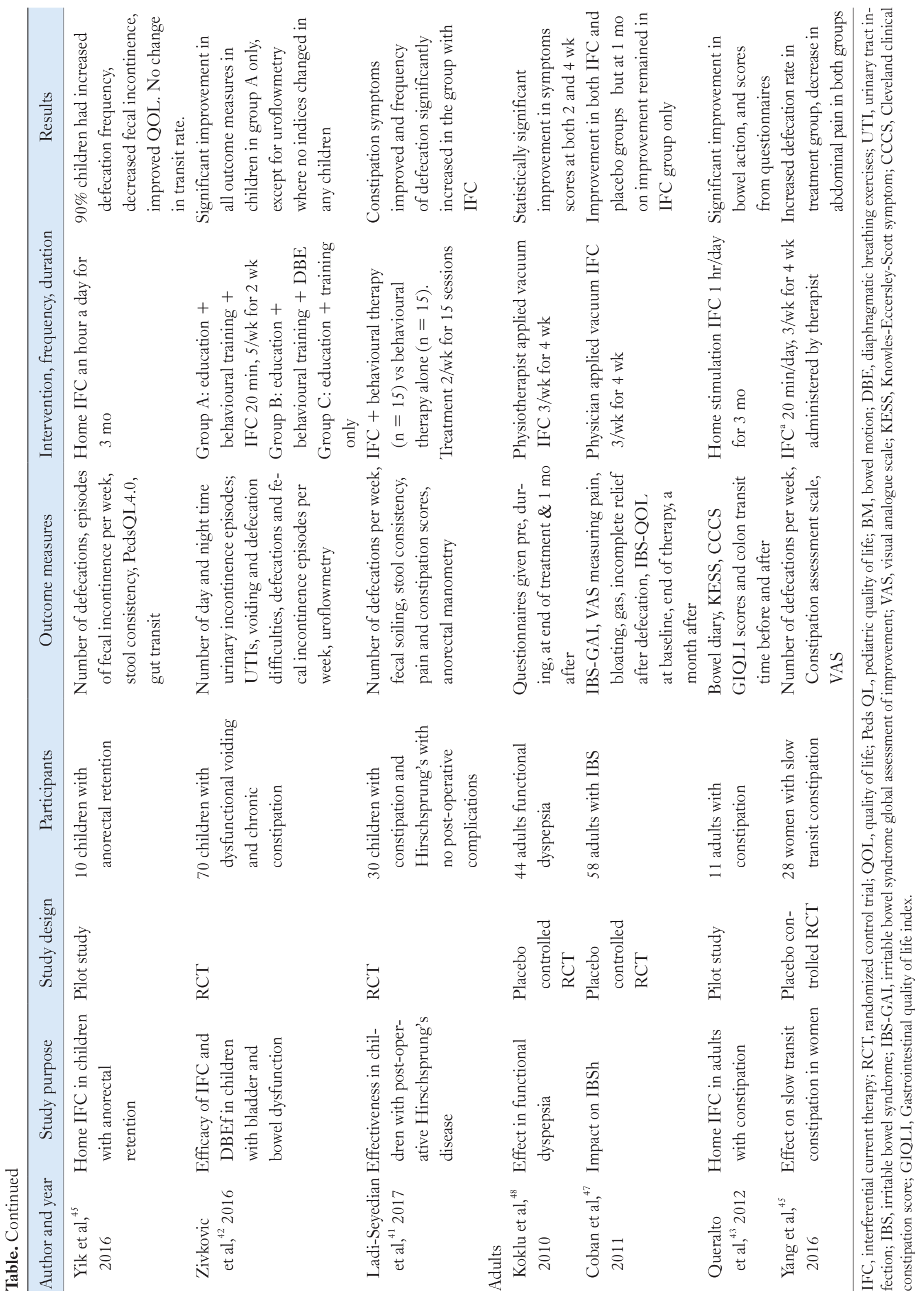


controlled studies are needed. ${ }^{51}$ While this is true, it unfortunately means that children in the placebo arm face the prospect of lack of effective therapy for some time, which potentially impacts on their future well-being from the social and developmental ramifications of constipation and fecal incontinence. ${ }^{37}$ It may be, therefore, preferable to keep the placebo-controlled studies confined to adults.

The theory that interferential current therapy works by creating a third therapeutic current at the point of bisection is appealing in that it targets places that have previously been relatively inaccessible. However, exactly what happens is hotly debated. ${ }^{18}$ The heterogeneity of skin, muscle and other tissue means an uneven resistance to electrical currents reducing the predictability of penetration of the interferential current. ${ }^{19}$ Nevertheless, numerous studies have found it to have benefit with several studies using a placebo-controlled group. This is difficult to accomplish as sham stimulation so far has been applied via no current passing between electrodes with the participant being told that the current is subsensory. ${ }^{23,38}$ This may convince children, but is less likely to do so in adults. These studies also required participants to come in to the clinic for treatment, which also makes it easier for an investigator to create a realistic sham scenario, whereas more recent devices are small enough for patients to take home and use. ${ }^{36}$ This also has implications for a sham control by this means. The moving of electrodes away from the therapeutic target and non-crossing of currents have been suggested as possible sham, but, as the actual predictability of current behaviour in human tissue is still unknown, it is possible some therapeutic benefit may occur.

Determining an appropriate sham is difficult as the exact mechanism of action of interferential current therapy is not known. However, the fact propagating sequences increased in a small group of children after treatment does suggest it may impact on the enteric nervous system potentially affecting overall neuronal health. ${ }^{37}$ This theory was postulated by the fact that effects took some time to develop, and lasted long after therapy had been ceased. As mentioned earlier, it is possible interferential current therapy stimulates motor and sensory spinal nerves, and/or the parasympathetic and sympathetic nerves, the interstitial cells of Cajal and/or other enteric nerves within the bowel wall. ${ }^{36}$ Means of identifying the exact mechanisms of action have yet to be developed.

In addition, as this is a novel means of treating gastrointestinal dysfunction the optimal stimulation parameters have not been determined. Nevertheless, several studies have used the same frequencies and device (EPM IF 4160 Fuji Dynamics Ltd, Kowloon, Hong Kong). ${ }^{52}$ In these studies, a $4-\mathrm{kHz}$ carrier frequency is selected with a beat frequency sweep of $80-160 \mathrm{~Hz}$ with a variable intensity of
$<33 \mathrm{~mA}$ in adults. The beat frequency sweep varied in pediatric studies from $80-120 \mathrm{~Hz}$ to $80-150 \mathrm{~Hz}$, $^{9,33,35}$

To date, no serious adverse effects have been described. The only reported side effect is diarrhea, which was the trigger for investigating its application in constipation in the first place. However, the effect of an electrical current on a developing fetus is unknown, and as such it is recommended that pregnancy or the desire to conceive be a contraindication. It should also not be used in the presence of a cardiac pacemaker ${ }^{44}$ as it may interfere with the programed therapeutic electrical activity. Neither should it be used in the presence of metal implants within the abdomen nor spine because of a potential risk of thermal injury. ${ }^{30}$ Caution should be applied.

\section{Conclusion}

There is speculative evidence that interferential current therapy is a viable alternative in reducing symptoms of constipation and fecal incontinence. While preliminary data in children is encouraging, more adult studies are needed, particularly placebo-controlled studies. Furthermore, an effective means of delivering a placebo current still needs to be identified, as comprehension on the exact distribution and effects on deeper tissue is still limited. ${ }^{19}$ Nevertheless interferential stimulation is an attractive therapy for chronic gastrointestinal disorders as it appears effective, is relatively low cost, and is a non-invasive and non-pharmacological intervention. ${ }^{13}$

\section{Financial support: None.}

\section{Conflicts of interest: None.}

Author contributions: Judith S Moore wrote the manuscript; and Peter R Gibson and Rebecca E Burgell were involved in the revision and approval of its final form for publication.

\section{References}

1. Velasco F. Neuromodulation: an overview. Arch Med Res 2000;31:232236.

2. Falletto E, Ganio E, Naldini G, Ratto C, Altomare DF. Sacral neuromodulation for bowel dysfunction: a consensus statement from the Italian group. Tech Coloproctol 2014;18:53-64.

3. Carrington EV, Evers J, Grossi U, et al. A systematic review of sacral nerve stimulation mechanisms in the treatment of fecal incontinence and constipation. Neurogastroenterol Motil 2014;26:1222-1237.

4. Jarrett ME, Mowatt G, Glazener CM, et al. Systematic review of sacral 
nerve stimulation for faecal incontinence and constipation. Br J Surg 2004;91:1559-1569.

5. Dinning PG, Scott SM. Novel diagnostics and therapy of colonic motor disorders. Curr Opin Pharmacol 2011;11:624-629.

6. Thin NN, Taylor SJ, Bremner SA, et al. Randomized clinical trial of sacral versus percutaneous tibial nerve stimulation in patients with faecal incontinence. Br J Surg 2015;102:349-358.

7. George AT, Dudding TC, Nicholls RJ, Vaizey CJ. A new minimally invasive technique for pudendal nerve stimulation. Colorectal Dis 2012;14:98-103.

8. Hotouras A, Thaha MA, Allison ME, Currie A, Scott SM, Chan CL. Percutaneous tibial nerve stimulation (PTNS) in females with faecal incontinence: the impact of sphincter morphology and rectal sensation on the clinical outcome. Int J Colorectal Dis 2012;27:927-930.

9. Chase J, Robertson VJ, Southwell B, Hutson J, Gibb S. Pilot study using transcutaneous electrical stimulation (interferential current) to treat chronic treatment-resistant constipation and soiling in children. J Gastroenterol Hepatol 2005;20:1054-1061.

10. Lee KJ, Kim JH, Cho SW. Short-term effects of magnetic sacral dermatome stimulation for idiopathic slow transit constipation: sham-controlled, cross-over pilot study. J Gastroenterol Hepatol 2006;21(1 pt 1):47-53.

11. Fuentes J, Armijo-Olivo S, Funabashi M, et al. Enhanced therapeutic alliance modulates pain intensity and muscle pain sensitivity in patients with chronic low back pain: an experimental controlled study. Phys Ther 2014;94:477-489.

12. Koca I, Boyaci A, Tutoglu A, Ucar M, Kocaturk O. Assessment of the effectiveness of interferential current therapy and TENS in the management of carpal tunnel syndrome: a randomized controlled study. Rheumatol Int 2014;34:1639-1645.

13. Hutson JM, Dughetti L, Stathopoulos L, Southwell BR. Transabdominal electrical stimulation (TES) for the treatment of slow-transit constipation (STC). Pediatr Surg Int 2015;31:445-4451.

14. Goats GC. Interferential current therapy. Br J Sports Med 1990;24:8792.

15. Ozcan J, Ward AR, Robertson VJ. A comparison of true and premodulated interferential currents. Arch Phys Med Rehabil 2004;85:409-415.

16. Agharezaee M, Mahnam A. A computational study to evaluate the activation pattern of nerve fibers in response to interferential currents stimulation. Med Biol Eng Comput 2015;53:713-720.

17. Palmer ST, Martin DJ, Steedman WM, Ravey J. Alteration of interferential current and transcutaneous electrical nerve stimulation frequency: effects on nerve excitation. Arch Phys Med Rehabil 1999;80:1065-1071.

18. Fiori A, Cescon CLC, Galesky JDF, Santos TACD, Brancalhão RMC, Bertolini GRF. Comparison between bipolar and tetrapolar of the interferential current in nociceptive threshold, accommodation and pleasantness in healthy individuals. Eur J Physiother 2014;16:201-205.

19. Beatti A, Rayner A, Chipchase L, Souvlis T. Penetration and spread of interferential current in cutaneous, subcutaneous and muscle tissues. Physiotherapy 2011;97:319-326.

20. Suh HR, Han HC, Cho HY. Immediate therapeutic effect of interferential current therapy on spasticity, balance, and gait function in chronic stroke patients: a randomized control trial. Clin Rehabil 2014;28:885-
891

21. Ward AR. Electrical stimulation using kilohertz-frequency alternating current. Phys Ther 2009;89:181-190.

22. Southwell BR, Yik YI, Tan A, Jordan-Ely J, Hutson JM. Transcutaneous electrical stimulation over the belly in slow-transit constipation. In: Nunez R, Fabbro MA, editors. Constipation in children: diagnosis and treatment. Hauppause: Nova Science Publishers Inc. 2013.

23. Clarke MC, Chase JW, Gibb S, et al. Decreased colonic transit time after transcutaneous interferential electrical stimulation in children with slow transit constipation. J Pediatr Surg 2009;44:408-412.

24. Brierley SM, Linden DR. Neuroplasticity and dysfunction after gastrointestinal inflammation. Nat Rev Gastroenterol Hepatol 2014;11:611627.

25. Van Poppel H, Ketelaer P, Van DeWeerd A. Interferential therapy for detrusor hyperreflexia in multiple sclerosis. Urology 1985;25:607-612.

26. Fuentes CJ, Armijo-Olivo S, Magee DJ, Gross DP. A preliminary investigation into the effects of active interferential current therapy and placebo on pressure pain sensitivity: a random crossover placebo controlled study. Physiotherapy 2011;97:291-301.

27. Keramat KU, Gaughran A. An unusual effect of interferential therapy. BMJ Case Rep Published Online First: 27 Jul 2012. doi: 10.1136/bcr2012-007648.

28. Elnaggar RK, Elshafey MA. Effects of combined resistive underwater exercises and interferential current therapy in patients with juvenile idiopathic arthritis: a randomized controlled trial. Am J Phys Med Rehabil 2016;95:96-102.

29. Beatti A, Tucker K, Chipchase LS. A double-blind placebo-controlled investigation into the effects of interferential therapy on experimentally induced pain using a cross-over design. Int Musculoskelet Med 2012;34:115-122.

30. Suriya-amarit D, Gaogasigam C, Siriphorn A, Boonyong S. Effect of interferential current stimulation in management of hemiplegic shoulder pain. Arch Phys Med Rehabil 2014;95:1441-1446.

31. Lee HE, Park K. Efficacy of salvage interferential electrical stimulation therapy in patients with medication-refractory enuresis: a pilot study. Int Neurourol J 2013;17:139-144.

32. Oh-Oka H. Efficacy of interferential low frequency therapy for elderly wet overactive bladder patients. Indian J Urol 2008;24:178-181.

33. Clarke MC, Catto-Smith AG, King SK, et al. Transabdominal electrical stimulation increases colonic propagating pressure waves in paediatric slow transit constipation. J Pediatr Surg 2012;47:2279-2284.

34. Yik YI, Clarke MC, Catto-Smith AG, et al. Slow-transit constipation with concurrent upper gastrointestinal dysmotility and its response to transcutaneous electrical stimulation. Pediatr Surg Int 2011;27:705-711.

35. Ismail KA, Chase J, Gibb S, et al. Daily transabdominal electrical stimulation at home increased defecation in children with slow-transit constipation: a pilot study. J Pediatr Surg 2009;44:2388-2392.

36. Yik YI, Ismail KA, Hutson JM, Southwell BR. Home transcutaneous electrical stimulation to treat children with slow-transit constipation. J Pediatr Surg 2012;47:1285-1290.

37. Clarke MC, Chase JW, Gibb S, Hutson JM, Southwell BR. Improvement of quality of life in children with slow transit constipation after 
treatment with transcutaneous electrical stimulation. J Pediatr Surg 2009;44:1268-1272.

38. Kajbafzadeh AM, Sharifi-Rad L, Nejat F, Kajbafzadeh M, Talaei HR. Transcutaneous interferential electrical stimulation for management of neurogenic bowel dysfunction in children with myelomeningocele. Int J Colorectal Dis 2012;27:453-458.

39. Lee YY, Erdogan A, Rao SS. High resolution and high definition anorectal manometry and pressure topography: diagnostic advance or a new kid on the block? Curr Gastroenterol Rep 2013;15:360.

40. Yik YI, Stathopoulos L, Hutson JM, Southwell BR. Home transcutaneous electrical stimulation to treat children with anorectal retention: a pilot study. Neuromodulation 2016;19:515-521.

41. Ladi-Seyedian SS, Sharifi-Rad L, Manouchehri N, Ashjaei B. A comparative study of transcutaneous interferential electrical stimulation plus behavioral therapy and behavioral therapy alone on constipation in postoperative Hirschsprung disease children. J Pediatr Surg 2017;52:177183.

42. Zivkovic VD, Stankovic I, Dimitrijevic L, et al. Are interferential electrical stimulation and diaphragmatic breathing exercises beneficial in children with bladder and bowel dysfunction? Urology 2016;102:207-212.

43. Queralto M, Vitton V, Bouvier M, Abysique A, Portier G. Interferential therapy: a new treatment for slow transit constipation. A pilot study in adults. Colorectal Dis 2013;15:e35-e39.

44. Vitton V, Benezech A, Honoré S, et al. CON-COUR study: interferential therapy in the treatment of chronic constipation in adults: study protocol for a randomized controlled trial. Trials 2015;16:234.

45. Yang Y, Yim J, Choi W, Lee S. Improving slow-transit constipation with transcutaneous electrical stimulation in women: a randomized, comparative study. Women Health 2017;57:494-507.

46. Drossman DA. Functional gastrointestinal disorders: history, pathophysiology, clinical features and rome IV. Gastroenterology 2016;150:1262$1279, \mathrm{e} 2$.

47. Coban Ş, Akbal E, Köklü S, et al. Clinical trial: transcutaneous interferential electrical stimulation in individuals with irritable bowel syndrome a prospective double-blind randomized study. Digestion 2012;86:86-93.

48. Köklü S, Köklü G, Ozgüçlü E, Kayani GU, Akbal E, Hasçelik Z. Clinical trial: interferential electric stimulation in functional dyspepsia patients a prospective randomized study. Aliment Pharmacol Ther 2010;31:961968.

49. Iqbal F, Thomas GP, Tan E, et al. Transcutaneous sacral electrical stimulation for chronic functional constipation. Dis Colon Rectum 2016;59:132-139.

50. Leong LC, Yik YI, Catto-Smith AG, Robertson VJ, Hutson JM, Southwell BR. Long-term effects of transabdominal electrical stimulation in treating children with slow-transit constipation. J Pediatr Surg 2011;46:2309-2312.

51. Lu ML, He J, Lu S. Electrical stimulation therapy for slow transit constipation in children: a systematic review. Int J Colorectal Dis 2015;30:697-702.

52. Yik YI, Leong LC, Hutson JM, Southwell BR. The impact of transcutaneous electrical stimulation on appendicostomy operation rates for children with chronic constipation - a single institution experience. J Pediatr Surg 2012;47:1421-1426. 